\title{
Práticas de gestão do conhecimento: caso dos sítios associados ao portal corporativo da FIOCRUZ
}

Danúzia da Rocha de Paula

\author{
Bibliotecária e Documentalista - Centro Latino \\ Americano de Estudos de Violência e Saúde Jorge \\ Careli, CLAVES/FIOCRUZ.
}

Regina de Barros Cianconi

Dra. em Ciência da Informação, Prof. Adjunta Universidade Federal Fluminense- UFF

Mapeamento das iniciativas de Gestão do Conhecimento disponíveis em sítios abrigados no Portal Fiocruz. A partir de revisão da literatura, entrevistas e questionários, foram identificadas práticas de Gestão do Conhecimento em sítios abrigados no portal corporativo da Fiocruz, que podem ser relevantes para a elaboração de um programa de Gestão do Conhecimento na instituição. Enfatiza que a implementação do portal corporativo cria ambiente de produtividade, viabilizando disseminação da informação, integração de processos, informações e pessoas, possibilitando troca de experiências e inovação.

Palavras-chave: Gestão do conhecimento; Gestão da informação; Melhores práticas; Portal corporativo; Fiocruz.

\section{Practices of knowledge management: case of the sites associated to the FIOCRUZ corporate portal}

Mapping of the Knowledge Management initiatives available on sites sheltered at Fiocruz Portal. By means of literature review, interviews and questionnaires, there were identified that some small sites at Fiocruz corporate portal have several knowledge management practices, that can be useful for the elaboration of a program of Knowledge Management in the institution. It shows that the implementation of a corporate portal allows a 
productivity environment, information dissemination, integration of processes, information and people, experience exchange and innovation.

Key-words: Knowledge Management; Information management; Best practices; Corporate portal; Fiocruz.

Recebido em 12.03.2007 Aceito em 20.05.2007

\section{Introdução}

As instituições governamentais produzem muitas informações de interesse público, mas nem sempre as organizam, disponibilizam e disseminam adequadamente. Assim como, poucos são os estímulos ao compartilhamento de experiências e ao aprendizado, de modo a facilitar a criação do conhecimento. Merecem destaque, portanto, as iniciativas que favorecem a organização, o acesso e o uso da informação.

No Brasil, implementam-se as diretrizes do Governo Eletrônico (egov $)^{1}$ com a intenção de utilizar as novas tecnologias de informação, em especial a Internet, na prestação de serviços e informações para os cidadãos.

O Governo Eletrônico expressa uma estratégia pela qual o aparelho do Estado faz uso das novas tecnologias para oferecer à sociedade melhores condições de acesso à informação e serviços governamentais, ampliando a qualidade desses serviços e garantindo maiores oportunidades de participação social no processo democrático (JARDIM, 2000).

O e-gov vem ao encontro da necessidade de organizar e disseminar informações e compartilhar conhecimento, na medida em que almeja a articulação de informações entre a sociedade e seu organizador. Neste processo, a disseminação da informação e a criação do conhecimento são importantes, e estas ações precisam de gestão.

A gestão do conhecimento é vista como elemento propulsor de mudanças na cultura organizacional e no comportamento dos indivíduos, a partir do estímulo ao compartilhamento intensivo de experiências e competências e do gerenciamento dos estoques informacionais, visando a criação de conhecimentos que subsidiem as ações organizacionais para garantir sua sobrevivência dentro dos parâmetros atuais (REIS, 2000) ${ }^{2}$.

A Fundação Oswaldo Cruz (Fiocruz) ${ }^{3}$, como uma instituição pública, procura seguir as diretrizes do governo eletrônico. Foi criada em 25 de

1 O Governo Eletrônico (e-gov) brasileiro, criado em 2000, é atribuição do Comitê Executivo do Governo Eletrônico (CEGE), com oito comitês técnicos para apoiar suas diretrizes, sendo presidido pelo Chefe da Casa Civil da Presidência da República.

2 Maria Elisa Andries dos Reis é Mestre em Gestão da Informação e Comunicação em Saúde, pela Fiocruz, tendo defendido dissertação sobre Portais Corporativos em 2005.

3 Mais informações sobre a Fiocruz no endereço: <http://www.fiocruz.br/>. Data de acesso: 09 jul. 2007. 
maio de 1900, com o nome de Instituto Soroterápico Federal, tendo como objetivo fabricar soros e vacinas contra a peste. Em pouco tempo, de simples produtor, passou a desenvolver pesquisa e medicina experimental. Em 1902, Oswaldo Cruz (Sanitarista) assumiu a presidência. Em 1908, o instituto foi rebatizado com o nome Instituto Oswaldo Cruz e, atendendo às solicitações do governo, seus pesquisadores realizaram expedições científicas nacionais e internacionais. Atualmente, com 106 anos, está vinculada ao Ministério da Saúde, sendo considerada a melhor instituição de Saúde Pública da América Latina, onde ocupa lugar de referência em ciência, tecnologia e em saúde.

O Portal da Fiocruz teve início em 2005, encontrando-se ainda em fase de estruturação. É orientado pelas diretrizes do Governo Eletrônico (e-gov), pelas determinações da lei de acessibilidade no 10.098 , e pelos princípios de usabilidade e navegabilidade, de acordo com a política de informação da Fiocruz.

Embora a Fiocruz não possua um Programa de Gestão do Conhecimento, existem várias práticas implementadas em seus sítios, que podem ser consideradas como relevantes no caso da implantação formal de uma política de gestão do conhecimento. Dentro deste contexto, tornase importante mapear as iniciativas de gestão do conhecimento existentes e disponíveis por meio dos diversos sítios que compõem o Portal Fiocruz.

Assim, foi realizado estudo em sítios setoriais da Fiocruz, com o objetivo de identificar os recursos e as práticas consideradas como de gestão do conhecimento que se encontrem suficientemente amadurecidas. De forma tal, que possam ser vistas como "melhores práticas" ${ }^{4}$, para serem candidatas à inclusão, de modo padronizado e integrado, no Portal Corporativo da instituição, em construção.

Para realizar a pesquisa, foi efetuada ampla revisão de literatura, buscando conceituar os temas envolvidos, descrever a evolução dos portais públicos e corporativos e sua inserção na política de informações do Governo brasileiro, especificamente nas políticas do Governo Eletrônico. Também foi realizada pesquisa em documentos da Fiocruz, que descrevem as diretrizes do seu Portal.

Em seguida, foi efetuada pesquisa de campo em dois sítios setoriais. $O$ instrumento de coleta de dados teve por base um trabalho de pesquisa, realizado para o Governo Federal, financiado pelo Instituto de Pesquisas Econômica e Aplicada - IPEA. O grupo de pesquisa desenvolveu metodologia, visando mensurar o grau de maturação das práticas de gestão do conhecimento na Administração Pública ${ }^{5}$. A referida

\footnotetext{
${ }^{4}$ Melhores práticas (best practices) é a identificação e difusão de casos de sucessos. Pode ser definido como um procedimento validado para a realização de uma tarefa ou solução de um problema. Inclui o contexto onde pode ser aplicado. São documentadas através de bancos de dados, manuais ou diretrizes.

$5 \mathrm{Na}$ referida pesquisa: "O governo que aprende - a gestão do conhecimento no setor público", financiada com recursos do Rede-IPEA, coordenada por Fábio Ferreira Batista, foram analisadas as mudanças que estão ocorrendo na Gestão Pública, no que se refere à implantação de práticas de Gestão do Conhecimento (GC) em 28
} 
metodologia, que se encontra disponível na Internet ${ }^{6}$, consiste de diversas assertivas. Cada uma deve ser analisada sob os aspectos de seu grau de amadurecimento e alcance na organização. Para desenvolvê-la, foram reunidos especialistas, que elaboraram um formulário extenso com questões objetivas e dissertativas.

Por se tratar de metodologia desenvolvida e já testada no próprio governo, optou-se por utilizar sua descrição das práticas de gestão do conhecimento ${ }^{7}$. Foi adaptada para o objetivo do trabalho, qual seja, mapear as iniciativas de práticas de gestão do conhecimento, tornadas disponíveis aos funcionários, por meio dos sítios associados ao Portal Fiocruz. Assim, a parte da metodologia utilizada, foi a que se refere às iniciativas de GC. Buscando-se, no caso deste trabalho, identificar as que possuem visibilidade por meio dos sítios das unidades.

Como a Fiocruz fomenta várias pesquisas na área da saúde, e as unidades setoriais produzem um volume intenso de informações relevantes para a sociedade, foram selecionados para a análise, dois entre os 15 sítios setoriais da Fiocruz. São eles: Casa de Oswaldo Cruz (COC) ${ }^{8}$ e Centro de Informação Científica e Tecnológica (CICT) ${ }^{9}$. Foram escolhidos por serem os responsáveis pela divulgação e organização de grande parte das informações produzidas na Fiocruz. A COC é a unidade responsável pela preservação e resgate de toda a história e organização da informação da instituição. O CICT é o responsável pela divulgação da informação. Para realizar o mapeamento das práticas de gestão do conhecimento, foram entrevistados os gestores destes dois sítios.

A motivação e marco teórico para o estudo das iniciativas em GC, seu grau de amadurecimento e alcance, têm suas bases no Governo Eletrônico. E também, nos princípios da gestão da informação (GI) e da gestão o conhecimento (GC), uma vez que os portais corporativos desempenham papel relevante nas práticas de ambas.

A premissa foi que, a partir do mapeamento, seja possível encontrar práticas identificadas como já amadurecidas e bem sedimentadas, de

órgãos da Administração Direta e em seis empresas estatais do Executivo Federal Brasileiro. (BATISTA et al, 2005).

6 Disponível em: <http://www.ipea.gov.br/pub/td/2005/td 1095.pdf>. Data de acesso: 09 jul. 2007.

7 Conforme já explicitado, a metodologia adaptada para a pesquisa, originalmente, destinou-se a analisar a implementação de práticas de Gestão do Conhecimento (GC) no Setor Público. Para isto, foi elaborada descrição detalhada das práticas de Gestão do Conhecimento, que foram utilizadas no presente trabalho.

${ }^{8}$ Casa de Oswaldo Cruz (Disponível em: <http://www.coc.fiocruz.br>. Data de acesso: 09 jul. 2007). Criado em 1986, é um centro de pesquisa, documentação e informação, que integra a Fiocruz, dedicado à memória, à história das ciências biomédicas e da saúde pública e à educação e divulgação em ciência e saúde.

${ }^{9}$ Centro de Informações Científicas e Tecnológicas (Disponível em: <http://www.cict.fiocruz.br>. Data de acesso: 09 jul. 2007). Criado em 1986, é a unidade da Fiocruz responsável por formular políticas, desenvolver estratégias e executar ações de informação e comunicação no campo da ciência e tecnologia em saúde, que respondam às demandas da sociedade, do Sistema Único de Saúde e de outros órgãos governamentais. 
modo que sejam aproveitadas. E possam ser estendidas aos demais sítios, integrando-as no Portal Corporativo.

\section{Governo eletrônico (e-gov)}

A política de informação norteia caminhos e ações a serem realizadas e está associada ao Estado, que utiliza a informação como insumo estratégico para o desenvolvimento científico-tecnológico. A política de informação governamental, tem a intenção de estimular a disseminação da informação, reduzir a exclusão digital, além de garantir o aprendizado e o desenvolvimento do indivíduo na sociedade.

O Brasil carece de uma política de informação nacional de cunho geral, que trate da informação em todos os seus aspectos e busque sistematizar e organizar o caos informacional nos organismos de governo. Bem como, promover legislação unificada que regule questões como privacidade, direito de acesso, propriedade da informação, participação social, entre outros.

No final do século $X X$, no Brasil, tiveram início as discussões com vistas à inserção do país na Sociedade da Informação. Como conseqüência, em 2000, foi produzido o Livro Verde da Sociedade da Informação ${ }^{10}$. Este pode ser visto como um esforço para delinear uma Política Nacional de Informação, embora destacando as tecnologias digitais.

Ainda com o foco nas tecnologias digitais, no mesmo ano, foi criado o Governo Eletrônico (e-gov), que destaca a importância do acesso à informação, priorizando o estabelecimento de normas, o desenvolvimento de processos que permitam a padronização dos serviços, as interfaces amigáveis, e as soluções integradas. Com o objetivo de democratizar e disseminar informação, promovendo parcerias entre 0 governo e 0 cidadão, na promoção da cidadania e desenvolvimento. Em suas diretrizes consta que, entre os papéis do e-gov, estão a promoção, o uso, e a disseminação de práticas de gestão do conhecimento nas instituições públicas (BRASIL, 2002).

Desta forma, estimula e sistematiza a criação de Portais no Governo Federal. Além de buscar o acompanhamento das melhores práticas em gestão do conhecimento, no âmbito da Administração Pública Federal (BRASIL, 2004). As três diretrizes alinhadas à estratégia do Governo Eletrônico no âmbito da Administração Pública são:

a) A definição clara e objetiva de conceitos referência sobre os princípios afetos à aplicação da gestão do conhecimento no setor público;

10 O Livro Verde da Sociedade da Informação encontra-se disponível para download no endereço: <http://www.socinfo.org.br/livro verde>. Data de acesso: 09 jul. 2007. 
b) Sistematicamente identificar, acompanhar e compartilhar as melhores práticas em gestão do conhecimento entre os atores do Governo Eletrônico (governo e sociedade civil);

c) A gestão do conhecimento no setor público deve ser objeto de política específica no âmbito das políticas de Governo Eletrônico (BRASIL, 2004, p.18).

é...

A visão da gestão do conhecimento segundo o Governo Eletrônico

(...) um conjunto de processos sistematizados, articulados e intencionais, que governam as ações de criação, captação, armazenamento, tratamento, disseminação e utilização de conhecimentos, com o propósito de atingir objetivos institucionais. Portanto, a Gestão do Conhecimento, entendida como gestão pública do conhecimento, torna-se instrumento estratégico fundamental para o desenvolvimento da nação brasileira, em particular para a criação de novo perfil da função pública baseada em pressupostos éticos, com vistas à produção compartilhada e colaborativa da informação e do conhecimento, bem como à distinção clara entre o interesse público e o interesse individual (BRASIL.2004, p.7).

Das diretrizes citadas, merece destaque a de letra $b$, que tem como um dos focos a ação de diagnosticar as melhores práticas em Portais Corporativos. Justifica, assim, a relevância do mapeamento dos sítios que fazem parte do Portal Fiocruz, buscando identificar o estágio de amadurecimento de iniciativas de gestão do conhecimento.

Entre os processos preconizados pelo e-gov (BRASIL, 2004, p.7) como sendo de gestão do conhecimento, as ações de "captação", "armazenamento", "tratamento" e "disseminação" de conhecimento, podem ser vistas como conhecimento explícito (ou seja, informação) ${ }^{11}$. Devendo, portanto, serem submetidos às práticas de gestão da informação.

\section{Gestão da informação}

A informação é insumo importante no processo de tomada de decisão de uma empresa ou instituição. As informações internas e externas produzidas pelas organizações precisam ser gerenciadas. A gestão da informação consiste na gestão de processos, sistemas, tecnologias, conteúdos, envolvendo o mapeamento das necessidades de

\footnotetext{
${ }^{11}$ Enquanto as ações de "criação" e "utilização" referem-se ao conhecimento tácito.
} 
informação, a produção da informação, sua coleta, organização, disseminação e uso.

Para que o uso da informação traga benefícios, esta precisa ser filtrada e administrada em todo em seu ciclo, de modo que sua gestão resulte em informações de qualidade.

Para que a gestão [de informação] seja eficaz é necessário que se estabeleça um conjunto de políticas coerentes que possibilitem o fornecimento de informações relevantes, com qualidade suficiente, precisa, transmitida para o local certo, no tempo correto, com um custo apropriado e facilidades de acesso por parte dos utilizadores. (BRAGA, data?? apud REIS, 2005, p.98).

Segundo Cianconi (2003) um sistema de gerenciamento dos recursos informacionais deve ser implementado de forma interativa, em rede. $\mathrm{E}$ deve surgir baseado no negócio da empresa, englobando: informações técnicas, informações administrativas, acervo bibliográfico e informações informais.

A gestão da informação faz parte do processo mais geral de gestão do conhecimento e ambas estão intrinsecamente ligadas. A disseminação das informações através do Portal Corporativo é importante para a construção do conhecimento na empresa. $E$, a partir da visão de seu papel estratégico, realizada em comunhão com a gestão do conhecimento, pode contribuir para uma organização com informações padronizadas e estruturadas, disponíveis com qualidade para os usuários internos e externos.

\section{Gestão do conhecimento (GC)}

As organizações estão passando por profundas mudanças e o conhecimento nas organizações se tornou 0 bem mais valioso na atualidade. Ainda que não exista consenso quanto à sua definição, a GC se consolidou em meados dos anos 90 do século passado. Torna-se objeto de interesse das organizações governamentais e privadas.

(...) A gestão do conhecimento consiste em ações sistemáticas, baseadas em políticas e metodologias, apoiadas em grande parte, mas não somente, em tecnologia, que variam conforme a cultura da organização, visando facilitar criação do conhecimento e seu aproveitamento na inteligência organizacional. (CIANCONI, 2003, p.251)

$\mathrm{Na}$ criação do conhecimento organizacional a informação e o conhecimento estão relacionados. O conceito de informação refere-se a um fluxo de mensagens, enquanto, o conhecimento é criado a partir desse fluxo de informação, ancorado nas crenças e compromissos de seu detentor (NONAKA; TAKEUCHI,1997). 
O conhecimento explícito é objetivo. Pode ser encontrado nos livros, manuais, base de dados, arquivos, sendo fácil de ser codificado e transmitido. O conhecimento tácito é pessoal. gerado no intelecto de cada um, sendo específico para uma determinada situação. É difícil de ser codificado, sendo fruto das experiências.

A GI está, portanto, interligada com a GC, e ambas podem ser vistas como parte de um mesmo processo organizacional. (CIANCONI, 2003).

No entanto, o que se convenciona chamar de gestão do conhecimento são, muitas vezes, apenas as práticas que envolvem pessoas. E à gestão da informação são reservadas as práticas que envolvem informação registrada e processos.

A FIG. 1, a seguir, sintetiza tal interseção, mostrando que, enquanto a GI envolve informação (conhecimento explícito, registrado) e processos, a GC envolve pessoas (conhecimento tácito), além das informações registradas e processos.

\begin{tabular}{|c|c|}
\hline \multicolumn{2}{|c|}{ Informação + Processos } \\
\hline \multicolumn{2}{|c|}{$\begin{array}{l}\text { Gestão da Informação } \\
\text { (Conhecimento Explícito) }\end{array}$} \\
\hline $\begin{array}{l}\text { Foco no } \\
\text { mapeamento de } \\
\text { processos, na gestão } \\
\text { dos conteúdos } \\
\text { informacionais }\end{array}$ & $\begin{array}{l}\text { Foco na } \\
\text { organização, } \\
\text { monitoramento e } \\
\text { recuperação } \\
\text { da informação }\end{array}$ \\
\hline $\begin{array}{l}\text { Foco nas } \\
\text { comunidades e } \\
\text { relacionamentos }\end{array}$ & $\begin{array}{l}\text { Foco na aprendizagem } \\
\text { organizacional e inovação }\end{array}$ \\
\hline \multicolumn{2}{|c|}{$\begin{array}{l}\text { (Conhecimento Tácito) } \\
\text { Gestão do Conhecimento }\end{array}$} \\
\hline Pessoas + Infor & ão + Processos \\
\hline
\end{tabular}

Figura 1 - Interseção entre a Gestão da Informação e a Gestão do Conhecimento

Fonte: Cianconi (2003, p.157)

A GI abrange a informação e os processos informacionais, sendo voltada aos estoques registrados, seu planejamento, organização e recuperação. Já a GC, deve ter foco mais abrangente, por envolver pessoas, informação e processos, isto é, o conhecimento tácito e explícito. 
Precisa estar preocupada também com as comunidades e a aprendizagem organizacional.

Algumas instituições implementam a gestão do conhecimento de modo mais completo, outras utilizam algumas práticas apenas. Entre as práticas de GC mais utilizadas estão: Gestão por competências, Mapeamento do conhecimento organizacional, Comunidades de prática, Comunidades virtuais, Melhores práticas, Aprendizagem organizacional e Ensino a distância.

As ferramentas utilizadas nas práticas de gestão do conhecimento permitem a integração, disseminação, democratização das informações produzidas pela organização, destacando-se entre elas os portais corporativos.

\section{Os portais corporativos}

Com o advento das tecnologias da informação e da Internet destaca-se o ambiente virtual, onde volumes imensos de informações ficam disponíveis.

Um Portal é o primeiro ponto de acesso do usuário a um sítio, tendo duas características comuns e principais: a integração e o compartilhamento. É um ambiente de produtividade, integrando informações (conteúdos), processos e pessoas, podendo ser composto dos ambientes: Internet, Intranet, Extranet.

Nesse ambiente são utilizadas diversas ferramentas de integração e interatividade. É um meio de acesso integrado que oferece aos visitantes um ponto único de contato para o fornecimento de informações e de serviços on-line (GANT; GANT 2001apud SIMÃO e RODRIGUES, 2005).

Convém destacar que, embora muitos usem indiscriminadamente os termos Intranet e portal, estes representam conceitos distintos. Sua caracterização é importante como base para o entendimento do Portal Fiocruz, que a despeito da denominação "portal", possui mais características de uma intranet ${ }^{12}$. Pode-se afirmar que o que veio a ser chamado de Portal Corporativo, uma única porta de entrada para os sistemas da organização, é uma evolução da concepção de intranet, à qual se acrescenta a integração e a interatividade.

A Intranet é uma iniciativa que busca tirar proveito das potencialidades da Internet para reunir a informação interna de uma organização e disponibilizá-la aos empregados, recorrendo à utilização de links para os conteúdos. Não há integração de aplicações e os sítios são descentralizados (os endereços de URL são independentes), fazendo com que os indivíduos precisem utilizar inúmeras aplicações distintas para desenvolver seu trabalho. Os mecanismos de busca, quando existem, são simplificados, assim como o emprego de taxonomias e a gestão de conteúdos.

12 Diferenças estabelecidas com base na experiência das autoras e em textos de OLIVEIRA (2002), TERRA; GORDON (2002), SALDANHA (2004). 
O Portal, ao contrário, deve ser um ambiente em que todas as informações necessárias aos processos estão disponíveis numa aplicação que integra todas as outras disponíveis na organização. Se o funcionário desejar consultar ou atualizar os seus dados pessoais, marcar reuniões, consultar legislação, metodologias internas, comunicar-se com os colegas, enviar e receber e-mails, consultar bases de dados, não necessita de abrir e fechar aplicações. Tudo estará facilmente acessível através de janelas de um único portal, que também permite a customização ou personalização dos conteúdos. Há emprego de ferramentas de trabalho colaborativo (groupware), tais como e-mail, chat agenda, videoconferência, tudo com interface padronizada.

A gestão de conteúdos é feita por meio de ferramentas próprias, assim como a medição de acesso, que utiliza sistema integrado ao portal e não uma ferramenta independente como costuma ocorrer nas Intranets. $\mathrm{E}$ o portal, necessariamente, inclui ferramenta de busca avançada, com várias possibilidades de cruzamento entre as informações, bem como utiliza taxonomias complexas, tesauros, terminologias etc. .

A customização dos serviços e informações, com base no perfil dos usuários e o emprego de interfaces personalizáveis, também são características dos portais corporativos.

Assim como, o uso de recursos sofisticados de desenvolvimento ( $X M L, P 2 P, B 2 B$, etc) e de segurança, (firewall, assinatura digital, criptografia).

Um portal pode, ainda, agregar as informações de dentro (entre os departamentos) e fora da empresa (clientes, parceiros, fornecedores), otimizando os recursos, estabelecendo padrões e metodologias unificados, minimizando custos.

\section{O Portal Fiocruz}

Em 2005, com o apoio científico e tecnológico do Centro de Informações Científicas e Tecnológicas (CICT), foi lançado o Portal Fiocruz. Visa integrar as unidades e articular as diversas linhas de pesquisa da instituição, bem como serviços e produtos em um espaço virtual, permitindo uma navegação ágil e objetiva. A Fiocruz pretende explorar o ambiente virtual, disseminar informações na instituição, promover a cultura, apoiar a pesquisa e o ensino. Com a iniciativa da Unidade CICT, foi possível atender às diretrizes da política de informação da Fiocruz, tendo o sítio da instituição sido transformado e re-estruturado com a arquitetura e os componentes de um Portal. No ar há dois anos, encontrase ainda na fase de estruturação, sendo a porta de entrada Fiocruz na Internet.

O Portal Fiocruz está disponível em duas versões (Inglês e Espanhol), ambas na mesma fase de estruturação. Sua estrutura é composta por conteúdos móveis e fixos. Os móveis são atualizados diariamente e contemplam os temas: Serviços oferecidos; Notícias; Pesquisador; Usuário/Profissional de saúde; Estudante; Fale conosco. Os conteúdos fixos são muitos, referem-se às funções, serviços e informações 
da instituição. Estão listados em menus no lado esquerdo do Portal. Há ligações (links) para os sítios das unidades da Fiocruz, onde o responsável pela alimentação é o gestor/produtor do conteúdo da unidade.

No entanto, até o momento, a instituição ainda não possui um Portal integrado, havendo um conjunto de sítios formados por meio de dois servidores que hospedam as páginas das diretorias, unidades, departamentos, programas. As tecnologias utilizadas são desenvolvidas internamente. O software do Portal Fiocruz ainda não é utilizado por todos os 15 sítios, para gerenciar o conjunto de informações produzidas e veiculadas por todos os setores da instituição. E as URLs dos sítios estão ainda independentes, não havendo a necessária integração, que configura um Portal Corporativo.

\section{As iniciativas de gestão do conhecimento nos sítios da Fiocruz}

Os dois sítios investigados são, a seguir, identificadas como área A e área $\mathrm{B}$.

A área A apresenta um site com poucos recursos tecnológicos para a organização da informação e, na sua apresentação, seus menus são fixos e listados no lado esquerdo do tela. O sítio não apresenta muitas características e recursos de um Portal Corporativo, encontrando-se em construção. Porém, possui um serviço de informações para os alunos/usuários muito eficiente.

A área B apresenta um sítio bem desenvolvido. Sua estrutura segue as diretrizes propostas pelo Governo Eletrônico (e-gov). A atualização do site é realizada diariamente, apresenta menus fixos e móveis, oferece as informações relevantes da instituição de modo organizado e objetivo, e também, serviços aos usuários.

Contudo, os dois sítios possuem ferramentas e conteúdos próprios, não havendo padronização de terminologia, nem de design. A recuperação em ambos limita-se à busca básica, não havendo possibilidade de busca avançada. Porém, no portal principal (que agrega os demais sítios), a busca avançada encontra-se em teste.

$\mathrm{Na}$ área $\mathrm{B}$, das 20 práticas de gestão do conhecimento citadas na metodologia, e incluídas no formulário de levantamento, verificou-se que 16 delas são implementadas e estão obtendo resultados importantes. São elas: Mentoring ${ }^{13}$, Mapeamento do conhecimento, Ferramentas de colaboração como portais, Intranets e Extranets, Sistema de gestão por competências, Banco de competências individuais, Memória organizacional, Educação corporativa (EAD), Mensuração do capital intelectual, Narrativas, Sistemas de workflow, Gestão de conteúdo, Costumer Relationship Management (CRM), Balanced Scorecard

13 Mentoring é uma modalidade de gestão do desempenho na qual um especialista participante (mentor) modela as competências de um indivíduo ou grupo, observa e analisa o desempenho, e retroalimenta a execução das atividades do indivíduo ou grupo. 
(BSC),Decision Support System (DSS), Enterprise Resource Planning (ERP) e Key Performance Indicators (KPI).

$\mathrm{Na}$ área $\mathrm{A}$, apenas 6 das práticas estão implementados e obtendo resultados importantes. São elas: Comunidades de prática, Coaching ${ }^{14}$, Mapeamento do conhecimento, Ferramentas de colaboração como portais e intranets, Gestão de conteúdo e Data mining (mineração de dados).

Ou seja, na área B $80 \%$ das principais práticas de gestão do conhecimento estão implementadas, enquanto somente $30 \%$ das práticas de gestão do conhecimento estão implementadas da área $A$.

As iniciativas de GC citadas a seguir, foram aquelas consideradas em estágio avançado de amadurecimento, nos sítios analisados: Comunidades de prática; Mentoring; Coaching; Melhores práticas; Mapeamento ou auditoria de conhecimento; Ferramentas de colaboração como portais, intranets e extranets; Sistema de gestão por competências; Banco de competências individuais/Banco de Talentos/Páginas Amarelas; Memória organizacional/Lições aprendidas/Banco de conhecimentos; Educação Corporativa (EAD); Mensuração do capital intelectual/ Mensuração dos ativos intangíveis; Narrativas (Relatos Pessoais); Sistemas de Workflow (Acompanhamento do fluxo de trabalho); Gestão do Conteúdo (Tratamento da informação); Gestão Eletrônica da Informação (GED); Data mining.

As iniciativas Sistemas de workflow, Gestão do conteúdo e data mining são práticas importantes de apoio à gestão do conhecimento, voltadas também para o conhecimento explícito (informação registrada).

A atual ausência de comunicação entre o Portal e os sítios das unidades, impossibilita a fidelidade das informações disponíveis no conteúdo do Portal. Foi identificado que o Portal gerencia a informação no estilo feudalismo, conforme a conceituação de McGee e Prusak (1994), isto é, a informação é gerenciada como em "feudos" ou "ilhas". Os departamentos atuam de modo isolado nos sítios, sem nenhum compartilhamento ou integração.

Houve bastante colaboração dos entrevistados para a realização do trabalho. $\mathrm{O}$ assunto desperta interesse na instituição e isto indica que há espaço para a formalização de uma política de gestão do conhecimento. Embora os departamentos dos sítios pesquisados apresentem visões diferentes e estágios diferenciados.

Identificou-se que o termo gestão do conhecimento é conhecido pelos gestores dos sítios, mas ainda não foram discutidas amplamente a sua importância e suas práticas. O que demonstra pouca valorização do potencial da gestão do conhecimento.

Existe, ainda, falta de padronização no uso das ferramentas utilizadas pelo Portal e pelos sítios das unidades, que não se encontram integradas, o que é necessário, segundo Terra e Gordon (2002), para configurar um Portal Corporativo. Desta forma, a Fiocruz, embora possua

${ }^{14}$ Coaching é similar ao mentoring, mas o coaching não participa da execução das atividades. Faz parte do processo de orientação, apoio, diálogo e acompanhamento, alinhado às diretrizes estratégicas. Tem atividades ligadas ao planejamento. 
um Portal, este, em termos de utilização das ferramentas e integração das informações e processos, encontra-se em estágio compatível com uma Intranet. A inconsistência, a redundância de informações, a ausência de integração de processo no Portal Fiocruz e seus sítios associados, prejudicam o usuário na busca de informação.

\section{Considerações finais}

Mudanças são necessárias para que, efetivamente, o Portal Fiocruz cumpra o papel de Portal corporativo. Que é bem mais complexo do que uma simples Intranet, com mais sofisticação nas ferramentas e no tratamento dos conteúdos e, principalmente, com foco na alavancagem do negócio da organização. As URLs devem ser integradas, bem como as ferramentas de colaboração e as aplicações internas comuns a mais de uma unidade, havendo necessidade de focar mais os negócios. Ações estão em andamento para que o Portal seja uma porta de entrada da Fiocruz na intranet e Internet, integrando as unidades da instituição, suas atividades e ferramentas, garantindo desse modo, a qualidade de informação armazenada no Portal.

Com o presente trabalho espera-se estar contribuindo para esse objetivo, havendo a possibilidade de estendê-lo aos demais sítios do Portal Fiocruz. Seria importante destacar e aproveitar as iniciativas já existentes.

É necessária política explícita para apresentar o Portal Fiocruz como instrumento para as ações de gestão do conhecimento, que permite potencializar a inovação, a criatividade e o conhecimento organizacional.

Iniciativas de gestão do conhecimento e da informação, se tornadas disponíveis de modo integrado no Portal da Fiocruz, podem dinamizar o trabalho interno/ externo da instituição. Isto resultará em produtos e serviços consistentes e informações precisas para o usuário, bem como, em compartilhamento de experiências, motivação, inovação.

O mapeamento das práticas de gestão do conhecimento efetuado em dois dos sítios da Fiocruz, se aplicado aos demais sítios, pode contribuir para seu aproveitamento no novo Portal corporativo, em consonância com o que prega o Governo Eletrônico. Finalizado o levantamento, um dos próximos passos seria a identificação das metodologias e tecnologias utilizadas nas práticas mapeadas, visando estabelecer padrões.

O estudo mostrou a aplicabilidade da metodologia de Batista et al (2005), para identificação e avaliação do grau de maturidade de iniciativas de gestão do conhecimento, tornadas disponíveis através de portais.

E permitiu dar visibilidade para a necessidade de uma ação unificada de gestão do conhecimento na Fiocruz. Que poderia levar à elaboração de um Programa de Gestão do Conhecimento na Instituição, abrangendo informações, processos e pessoas. 


\section{Referências}

BATISTA, F. F. et al. Gestão do Conhecimento na administração pública. Brasília: Ipea, 2005. (Texto para discussão no 1095). Disponível em $<$ http://www.ipea.gov.br/pub/td/2005/td 1095.pdf>. Acesso em: 11 maio 2005.

BRASIL. Ministério da Saúde. Centro de Informação Científica e Tecnológica. Programa integrado de informação e comunicação da FIOCRUZ. Rio de Janeiro: FIOCRUZ, 2003. 29p.

BRASIL. Ministério do Planejamento. Oficinas de planejamento estratégico. Comitê Executivo do Governo Eletrônico. Comitês Técnicos, 2004. 23p. Relatórios Consolidados. Disponível em: <http//:www.governoeletronico.gov.br/governoeletronico> Acesso em: 04 jun. 2006.

CIANCONI, R. Gestão do conhecimento: visão de indivíduos e organizações no Brasil. Tese (Doutorado) - Escola de Comunicação / UFRJ, Rio de Janeiro, 2003.

SIMÃO, J. B; RODRIGUES, G. Acessibilidade às informações públicas: uma avaliação do portal de serviços e informações do Governo Federal. Revista Ciência da Informação, Brasília, v. 34, n. 2 maio/agos., 2005. Disponível em <www.scielo.br/scielo.php?pid= S010019652005000200009\&script=sci arttext >. Data de acesso: 05 jan. 2006.

JARDIM, J. M. Capacidade governativa, informação e governo eletrônico. DataGramaZero, Rio de Janeiro, v. 1, n. 5, p. 1-8. out. 2000. Disponível em: <http://www.dgz.org.br/out00/F I aut.htm>. Data de acesso: 04 jun. 2006.

McGEE, J.; PRUSAK, L. Gerenciamento estratégico da informação: aumente a competitividade e a eficiência de sua empresa utilizando a informação como uma ferramenta estratégica. Rio de Janeiro: Campus, 1994.

NONAKA, I; TAKEUUCHI, H. Criação do conhecimento na empresa: como as empresa japonesas geram a dinâmica da inovação. Rio de Janeiro: Campus; 1997.

OLIVEIRA, V. A. Portal Corporativo: o novo desktop do trabalhador. 2002. Disponível em:<http://www.tiadro.com/News/artigos/portalcorp.html>. Data de acesso: 18 jan. 2007.

REIS, M. E. A. Portal corporativo como ferramenta de gestão do conhecimento. Dissertação (Mestrado) - ENSP/FIOCRUZ, Rio de Janeiro, 2005.

SALDANHA, Ricardo. Portal corporativo: complexo e estratégico. 2004. Disponível em <http://www.intranetportal.com.br/estrategia/e2>. Data de acesso: 05 jan. 2007. 
SIMÃO, J. B; RODRIGUES, G. Acessibilidade às informações públicas: uma avaliação do portal de serviços e informações do Governo Federal. Revista Ciência da Informação, Brasília, v. 34, n. 2 maio/agos., 2005. Disponível em S010019652005000200009\&script=sci arttext >. Data de acesso: 05 jan. 2006. TERRA, J. C. C.; GORDON C. Portais corporativos: a revolução na gestão do conhecimento. São Paulo: Negócio Editora, 2002. 